\title{
ON IMPROVING DOPPLER ULTRASOUND SPECTROSCOPY WITH MULTIBAND INSTANTANEOUS ENERGY SEPARATION
}

\author{
Petros Maragos, Thanasis Loupas, and Vassilis Pitsikalis \\ Dept. of E.C.E., National Technical University of Athens, Zografou, Athens 15773, Greece. \\ E-mail: [maragos, vpitsik] @cs.ntua.gr, tloupasecentral.ntua.gr.
}

\begin{abstract}
This paper deals with improving the time-frequency resolution of Doppler ultrasound spectroscopy, applied to blood flow analysis, by developing robust nonstationary spectrum estimation techniques based on Gabor filterbanks and multiband AM-FM demodulation that uses an instantaneous energy separation algorithm.
\end{abstract}

\section{INTRODUCTION}

Spectral Doppler ultrasound represents an invaluable tool for the noninvasive measurement of blood flow, with extensive medical applications in the diagnosis and management of cardiovascular disease [6] . Spectral Doppler ultrasound systems operate by transmitting multiple ultrasonic pulses along a given beam direction, receiving the Doppler signal backscattered from moving blood cells at a given depth, and performing spectral analysis of the Doppler signal to produce a two-dimensional spectrogram which provides the amplitudes of the detected Doppler frequency shifts (proportional to the corresponding axial velocity components of the moving blood cells) as a function of time. In all commercially available systems, spectral analysis is carried out by means of the short-time Fourier transform. However, it is generally recognized that this type of conventional spectral analysis is associated with a number of errors in the context of quantitative blood flow measurements.

More specifically, a first source of error is the temporal windowing (typically, $20-30 \mathrm{~ms}$ ) associated with the shorttime Fourier transform, which causes artificial broadening of the true Doppler spectrum. A second source of error is introduced by the highly non-stationary nature of Doppler signals from blood, resulting in spectral distortion and generation of artificial frequency components. Obviously, an attempt to minimize the effect of temporal windowing by using a longer analysis window will accentuate the non-stationarityrelated distortion and vice versa. A third source of error is due to the amplitude modulation of the Doppler signal by

This work was supported by the basic research program ARCHIMEDES of the NTUA Institute of Communication and Computer Systems. the impulse response of the ultrasound system, which corresponds to implicit windowing in the spatial domain. The above sources of error, which have been collectively grouped under the term "spectral broadening artifacts", are considered responsible for limiting the performance and, in certain cases, diagnostic utility of spectral Doppler ultrasound. A quantitative model of spectral broadening can be found in [2], where as a detailed review is available in [11].

Motivated by the specific drawbacks of the short-time Fourier transform in the context of Doppler ultrasound, a number of alternative spectral analysis techniques have been proposed in the literature, including AR and ARMA models [17, 7, 9, 14], Wigner distributions [5, 10, 4], and MUSIC algorithms $[1,18]$. However, none of those alternative spectral analysis techniques have been adopted in routine clinical practice due to concerns about their compatibility with the non-stationary nature of blood flow (i.e., "optimum" analysis parameters vary widely throughout the cardiac cycle) and/or technique-specific artifacts (e.g. Wigner distribution frequency cross-products). Therefore, a considerable need still remains for new spectral analysis techniques which are compatible with the non-stationarity of Doppler signals from blood, and offer clearly better performance than the shorttime Fourier transform, both in terms of spectral broadening and distortion artifacts as well as temporal / frequency resolution.

A relatively new trend in nonstationary signal and spectrum analysis is to model a signal as a sum of AM-FM signals, i.e., nonstationary sines $x(t)=a(t) \cos \left(\int_{0}^{t} f(\tau) d \tau\right)$ that have a combined amplitude modulation (AM) and frequency modulation (FM). To solve the demodulation problem, an efficient novel methodology was developed in [15] by tracking the energy of the source producing the AM-FM oscillation and separating it into its instantaneous amplitude and frequency components. The energy tracking is done via nonlinear differential operators, the main representative of which is the continuous-time Teager-Kaiser energy operator [12] $\Psi[x(t)] \triangleq[\dot{x}(t)]^{2}-x(t) \ddot{x}(t)$, where $\dot{x}(t)=d x(t) / d t$. The demodulation is done via the energy separation algorithm (ESA) [15]. In this paper, we use novel tools such as a multiband version of the ESA [3] and related time-frequency 
robust estimates [16] to develop an improved method for Doppler ultrasound spectroscopy. We apply the new method to blood flow signals and compare it with traditional techniques.

\section{SIMULATED SIGNALS}

Two types of Doppler data were used as inputs in this study: 1) synthetic radiofrequency (RF) signals, generated according to a model which specified the signals' true spectral content; and 2) in vivo blood flow RF signals, which allowed testing and evaluation under realistic conditions.

The synthetic RF Doppler signals were obtained by means of a backscatter simulation model which convolved the ultrasound system's impulse response with a spatio-temporal distribution of multiple elementary targets according to the equation:

$$
r f(i, t)=\sum_{m=-M}^{M} \sum_{n=-N}^{N} h_{V}(m, n) * s\left(r_{i}+m, c_{i}+n, t\right)
$$

where $r, c$ represent the longitudinal and transverse axis of the simulated vessel and $r_{i}, c_{i}$ define the ultrasound beam axis, which forms an angle $\theta$ relative to the vessel's longitudinal axis: i.e., $r_{i}=r_{0}+(i-1) d r, \quad c_{i}=c_{0}+(i-1) d c, \theta=$ $\arctan (d c / d r)$. The function $r f(i, t)$ denotes the backscattered RF sample $i$ received at discrete time $t=0, T, 2 T, \ldots$ where $T$ is the pulse repetition period. The matrix $h_{V}(m, n)$ represents the ultrasound system's impulse response (first defined in coordinates parallel and perpendicular to the ultrasound beam, and then rotated to coincide with the vessel coordinates $r, c)$. The function $s(r, c, t)$ specifies the spatiotemporal distribution of backscatter targets simulating the red blood cells. The targets are randomly initialized in terms of their scattering strength, and undergo shifting according to the model

$$
\begin{aligned}
s(r, c, t) & =s(r+v(c, t) T, c, t-T) \\
v(c, t) & =v_{0}(t)\left(1-\frac{\left|c-c_{0}\right|^{k(t)}}{R^{k(t)}}\right)
\end{aligned}
$$

where $v_{0}(t)$ is the target velocity on the vessel longitudinal axis $c=c_{0}, R$ is the vessel radius, and $k(t)$ determines the blood velocity profile.

The developed simulation environment included GUIs which allowed full control of all the important instrumentationand physics- related parameters such as ultrasound pulse frequency and length, beam width, pulse repetition frequency, maximum velocity, beam-vessel angle, etc.

The in vivo blood flow RF signals were acquired from the common carotid artery, using a central pulse frequency of 4 $\mathrm{MHz}, \mathrm{RF}$ sampling frequency of $24 \mathrm{MHz}$, pulse repetition frequency of $5 \mathrm{kHz}$, beam-vessel angle of 60 degrees and observation time of 2 seconds.

\section{ENERGY SEPARATION ALGORITHM}

We assume that the original (possibly multicomponent) nonstationary signal under analysis has been filtered through a bank of bandpass filters. Let the output from a single filter be modeled as an AM-FM signal $x(t)=a(t) \cos \left(\int_{0}^{t} f(\tau) d \tau\right)$. Applying the energy operator $\Psi$ to this signal yields the instantaneous source energy, i.e. $\Psi[x(t)] \approx a^{2}(t) f^{2}(t)$, where the approximation error becomes negligible [15] if the instantaneous amplitude $a(t)$ and instantaneous frequency $f(t)$ do not vary too fast or too much with respect to the average value of $f(t)$. Then, AM-FM demodulation can be achieved by separating the instantaneous energy into its amplitude and frequency components. $\Psi$ is the main ingredient of the first energy separation algorithm (ESA)

$$
\sqrt{\frac{\Psi[x(t)]}{\Psi[\dot{x}(t)]}} \approx f(t) \quad, \quad \frac{\Psi[x(t)]}{\sqrt{\Psi[\dot{x}(t)]}} \approx|a(t)|
$$

developed in [15] and used for signal and speech AM-FM demodulation.

The instantaneous energy separation methodology has led to several classes of algorithms for demodulating discretetime AM-FM signals $x[n]=x(n T)=A[n] \cos \left(\int_{0}^{n} F[k] d k\right)$ where $A[n]=a(n T)$ and $F[n]=T f(n T)$. A direct approach is to apply the discrete-time Teager-Kaiser operator $\Psi_{d}\left[x_{n}\right] \triangleq x_{n}^{2}-x_{n-1} x_{n+1}$, where $x_{n}=x[n]$, to the discrete AM-FM signal and and its differences (which approximate derivatives). This yields the following algorithm, called Discrete ESA [15]:

$$
\begin{gathered}
\arccos \left(1-\frac{\Psi_{d}\left[x_{n}-x_{n-1}\right]+\Psi_{d}\left[x_{n+1}-x_{n}\right]}{4 \Psi_{d}\left[x_{n}\right]}\right) \approx F[n] \\
\sqrt{\frac{\Psi_{d}\left[x_{n}\right]}{\sin ^{2}(F[n])}} \approx|A[n]|
\end{gathered}
$$

The DESA is a novel and very promising approach to AMFM demodulation for many reasons: (i) It yields very small errors for estimating the instantaneous amplitude and frequency. (ii) It has an extremely low computational complexity. (iii) It has an excellent time resolution, almost instantaneous; i.e., operates on a 5-sample moving window and can track instantaneous changes of signal modulations. (iv) It is less computationally complex and has better time resolution than other classical demodulation approaches such as the Hilbert transform. (v) It can detect transient events.

\section{MULTIBAND ANALYSIS AND EXPERIMENTS}

In order to take advantage of ESA's capabilities, the doppler RF signals are filtered through a filter-bank constructed by real ${ }^{1}$ Gabor bandpass filters. Gabor filters [8] are known to be optimally compact and smooth both in time and frequency

\footnotetext{
${ }^{1}$ At this stage of the analysis no complex signals are used.
} 
domains and have impulse response $h(t)$ and frequency response $H(\omega)$,

$$
\begin{array}{r}
h(t)=e^{-\alpha^{2} t^{2}} \cos (2 \pi \nu t) \\
H(\omega)=\frac{\sqrt{\pi}}{2 \alpha}\left[e^{-\frac{\pi(\omega-\nu)^{2}}{\alpha^{2}}}+e^{-\frac{\pi(\omega+\nu)^{2}}{\alpha^{2}}}\right]
\end{array}
$$

where $\nu$ is the center frequency of each filter and $\alpha$ is the bandwidth parameter. (The rms bandwidth is equal to $\alpha / \sqrt{2 \pi}$.)

The bandpass signals produced as the outputs of a dense bank of overlapping uniformly-spaced Gabor filters are demodulated by using the ESA algorithm, thereby yielding instantaneous frequency $f(t)$ and amplitude $|a(t)|$ estimates for each filter output. For the AM-FM signal at each filter's output, a robust estimate of its short-time mean frequency, weighted by the squared amplitude $[a(t)]^{2}$, is given by [16]

$$
F_{w}=\frac{\int_{t_{0}}^{t_{0}+T} f(t)[a(t)]^{2} d t}{\int_{t_{0}}^{t_{0}+T}[a(t)]^{2} d t}
$$

for each analysis frame centered around time $t$ and each filter centered around a central frequency $\nu$. The denser the Gabor filterbank, the better the frequency resolution. Timeresolution depends on the length of the analysis frame. Typical durations of the analysis frame for this category of signals (Section 2) vary between 5-30 ms. The frequency steps between adjacent filters that have been used vary between 200-500 Hz. In Figs. 1(b) and 2(b) a time-frequency representation known as pyknogram [16] is shown as a binary image, in which for each analysis frame time center and for each filter's center frequency a dot is plotted at the weighted short-time mean frequency estimate $F_{w}$ (which in general may be different from the filter's center frequency). Since dense regions correspond to dominant frequencies at each time instant, the pyknogram is a useful tool for frequency estimation. Fig. 1(b) presents the pyknogram of a synthetic signal with constant flow velocity, constant velocity profile, central pulse frequency of $4 \mathrm{MHz}$, RF sampling frequency of $20 \mathrm{MHz}$, pulse repetition frequency of $8 \mathrm{kHz}$, beam-vessel angle of 45 degrees and observation time of $2 \mathrm{sec}$. In Fig. 2(b) the same method has been employed for the real part of the in vivo blood flow RF signal. For both experiments, we used moving frames of 128 samples with $50 \%$ overlap for computing $F_{w}$. It is evident that the pyknogram tracks the dominant frequencies throughout the duration of the two cardiac cycles. In the above mentioned pyknograms the lighter dots correspond to bandpass signals whose Teager energy [15] surpasses a threshold.

We have compared the above pyknogram method with the short-time Fourier transform (STFT) method for spectral estimation. In Figs. 1(c) and 2(c) the STFTs of the two signals are presented. (For computing the STFT, 256-point DFT's were used on moving Hamming-windowed frames of 128 samples with a $50 \%$ overlap.) From preliminary experiments, the pyknogram appears to offer a better spectral resolution than the STFT. A detailed quantitative evaluation of the two techniques is currently in progress.

\section{CONCLUSIONS}

In this paper multiband analysis and ESA [15, 3, 16] have been succesfully introduced as an attempt to improve doppler ultrasound spectroscopy. Our initial experience is clearly promising and our on-going research work in this area includes: extension of the multiband energy demodulation analysis for complex doppler signals, extended experiments and comparison with STFT and other alternative methods.

\section{REFERENCES}

[1] M.E. Allam, R.R. Kinnick, and J.F. Greenleaf, "Isomorphism between pulsed-Wave Doppler ultrasound and direction-ofarrival estimation. Part I: Basic principles; Part II: Experimental results", IEEE Trans. Ultrasonics, Ferroelectrics, \& Freq. Control, vol. 43, pp. 911-934, 1996.

[2] C.A.C. Bastos, R.J. Fish, and F. Vaz, "Spectrum of Doppler ultrasound signals from non-stationary blood flow", IEEE Trans. Ultrasonics, Ferroelectrics, \& Freq. Control, vol. 46, pp. 1201-1211, 1999.

[3] A. C. Bovik, P. Maragos, and T.F. Quatieri, "AM-FM Energy Detection and Separation in Noise Using Multiband Energy Operators", IEEE Trans. Signal Processing, vol. 41, Dec. 1993.

[4] J.C. Cardoso, M.G. Ruano, and P.J. Fish, "Nonstationarity broadening reduction in pulsed Doppler spectrum measurements using time-frequency estimators", IEEE Trans. Biomedical Engineering, vol. 43, pp. 1176-1186, 1996.

[5] J.Y. David, S.A. Jones, and D.P. Giddens, "Modern spectral analysis techniques for blood flow velocity and spectral measurements with pulsed Doppler ultrasound", IEEE Trans. Biomedical Engineering, vol. 38, pp. 589-596, 1991.

[6] D. H. Evans and W. N. McDicken, "Doppler Ultrasound: Physics, Instrumentation, and Signal Processing”, Wiley, 2000.

[7] F. Forsberg, "On the usefulness of singular value decomposition - ARMA models in Doppler ultrasound", IEEE Trans. Ultrasonics, Ferroelectrics, \& Freq. Control, vol. 38, pp. 418-428, 1993.

[8] D. Gabor, "Theory of communication", Journal of the Institution of Electrical Engineers, 93(III):429-457, 1946.

[9] N.F. Guler, et al., "Comparison of FFT-and AR-based sonogram outputs of $20 \mathrm{MHz}$ pulsed Doppler data in real time", Computers in Biology and Medicine, vol. 25, pp. 383-391, 1995. 


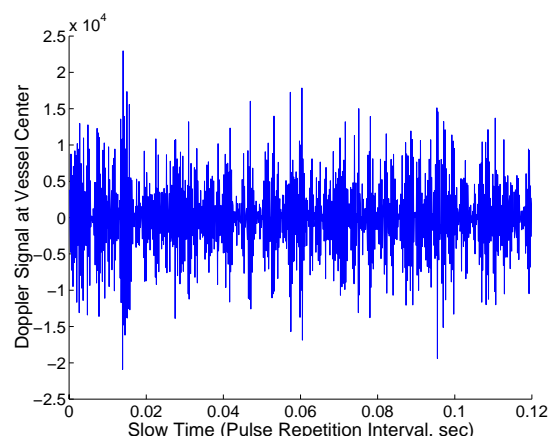

(a)

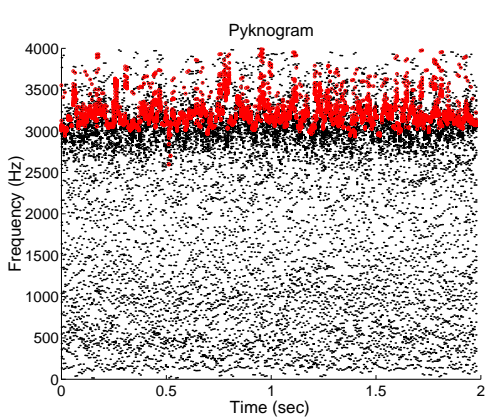

(b)

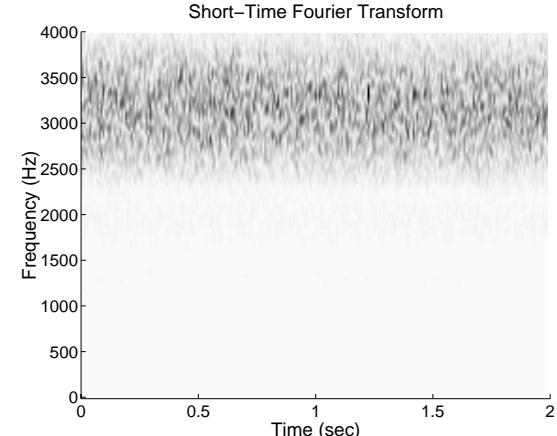

(c)

Fig. 1. (a) Synthetic Doppler Signal, (b) Pyknogram of Synthetic Signal, (c) STFT of Synthetic Signal.

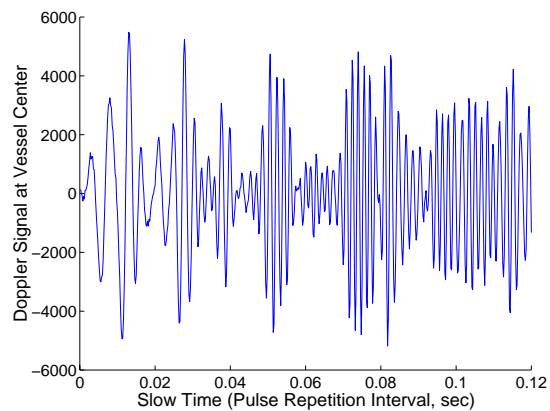

(a)

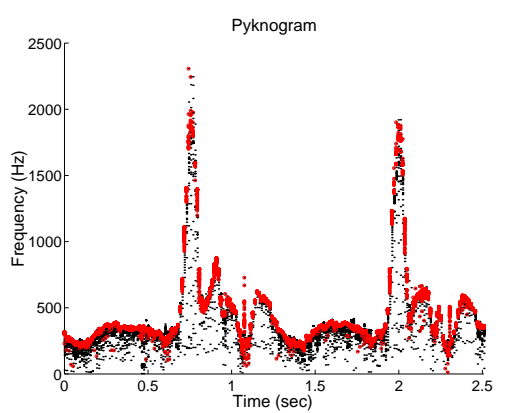

(b)

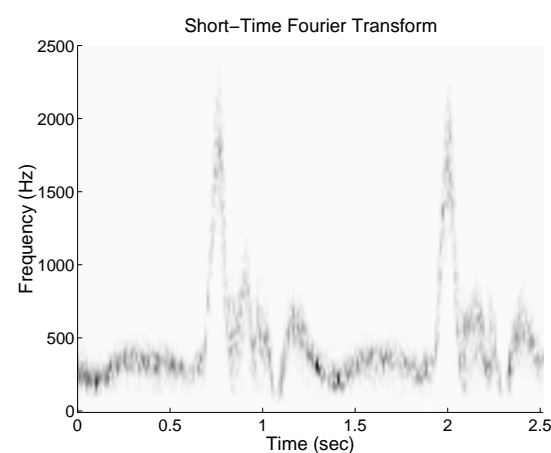

(c)

Fig. 2. (a) In vivo Doppler Signal, (b) Pyknogram of In vivo Signal, (c) STFT of In vivo Signal.

[10] Z. Guo, L.G. Durand, and H.C. Lee, "Comparison of timefrequency distribution techniques for analysis of simulated Doppler ultrasound signals of the femoral artery", IEEE Trans. Biomedical Engineering, vol. 41, pp. 332-342, 1994.

[11] S.A. Jones, "Fundamental sources of error and spectral broadening in Doppler ultrasound signals",Critical Reviews in Biomedical Engineering, vol. 21, pp. 399-483, 1993.

[12] J. F. Kaiser, "On a simple algorithm to calculate the 'energy' of a signal," Proc. ICASSP'90, Albuquerque, New Mexico, April 1990, pp. 381-384.

[13] J. F. Kaiser, "On Teager's energy algorithm and its generalization to continuous signals," Proc. IEEE Digital Signal Processing Workshop, Mohonk (New Paltz), New York, Sept. 1990.

[14] P.I. Keeton, et al, "Spectral broadening of clinical Doppler signals using FFT and autoregressive modeling", European Journal of Ultrasound, vol. 7, pp. 209-218, 1998.

[15] P. Maragos, J. F. Kaiser, and T. F. Quatieri, "Energy Separation in Signal Modulations with Application to Speech Analysis”, IEEE Trans. Signal Processing, vol. 41, pp. 3024-3051, Oct. 1993.
[16] A. Potamianos and P. Maragos, "Speech Formant Frequency and Bandwidth Tracking Using Multiband Energy Demodulation", J. Acoust. Soc. Amer., 99 (6), pp.3795-3806, June 1996.

[17] Vaitkus PJ, et al, "A comparative study and assessment of Doppler ultrasound spectral estimation techniques. Part I: Estimation methods; Part II: Methods and results", Ultrasound in Medicine and Biology, vol. 14, pp. 661-688, 1988.

[18] P.J. Vaitkus, R.S.C. Cobbold, and K.W. Johnston, "A new time-domain narrowband velocity estimation technique for Doppler ultrasound flow imaging. Part I: Theory; Part II: Comparative performance assessment", IEEE Trans. Ultrasonics, Ferroelectrics, \& Freq. Control, vol. 45, pp. 939-965, 1998. 